\title{
The Theory of TOROIDALLY CONFINED PLASMAS
}

Third Edition 
This page intentionally left blank 


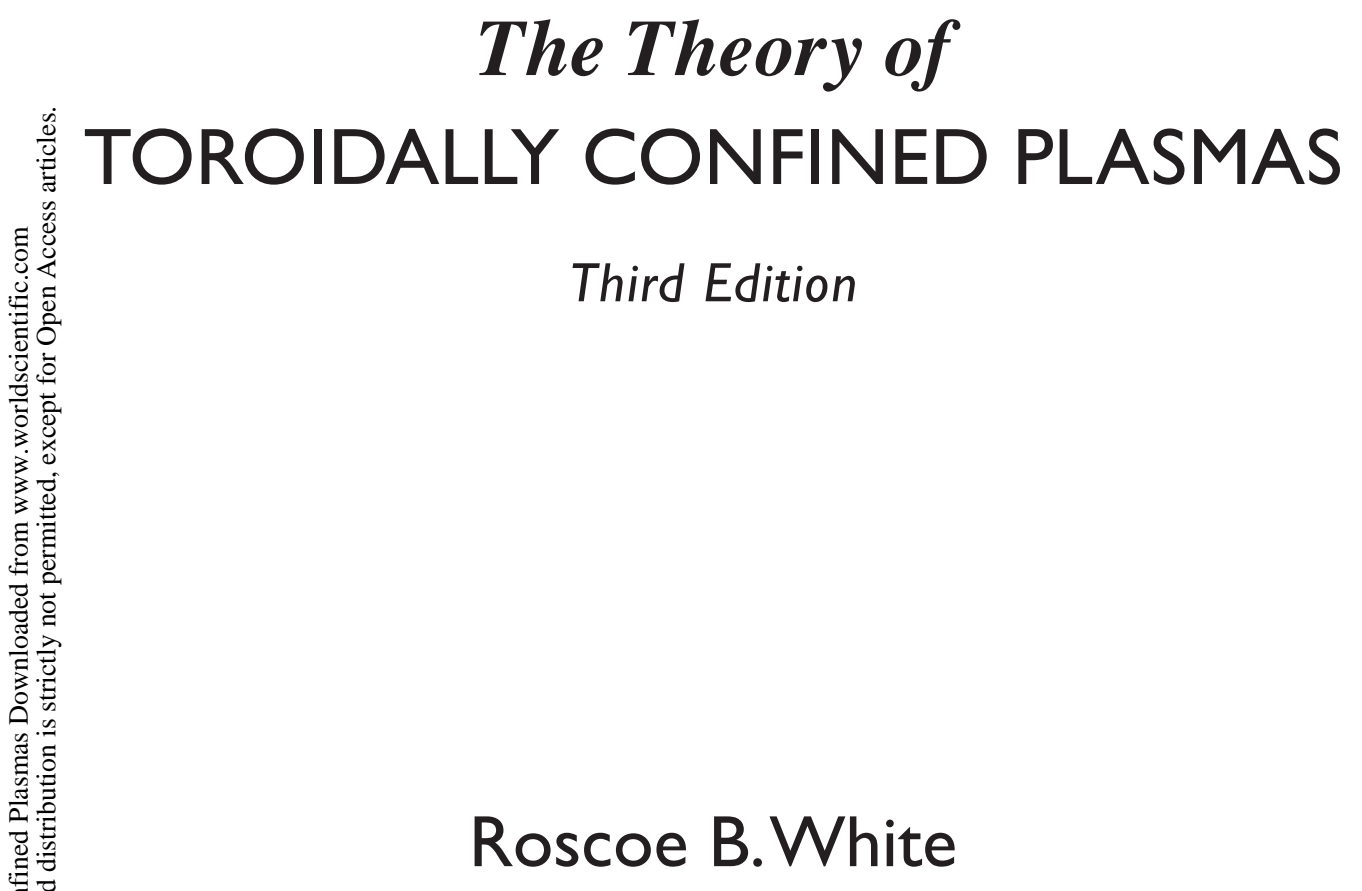

Princeton University 
Published by

Imperial College Press

57 Shelton Street

Covent Garden

London WC2H 9HE

\section{Distributed by}

World Scientific Publishing Co. Pte. Ltd.

5 Toh Tuck Link, Singapore 596224

USA office: 27 Warren Street, Suite 401-402, Hackensack, NJ 07601

UK office: 57 Shelton Street, Covent Garden, London WC2H 9HE

\section{British Library Cataloguing-in-Publication Data}

A catalogue record for this book is available from the British Library.

\section{THE THEORY OF TOROIDALLY CONFINED PLASMAS Third Edition}

Copyright $(C) 2014$ by Imperial College Press

All rights reserved. This book, or parts thereof, may not be reproduced in any form or by any means, electronic or mechanical, including photocopying, recording or any information storage and retrieval system now known or to be invented, without written permission from the Publisher.

For photocopying of material in this volume, please pay a copying fee through the Copyright Clearance Center, Inc., 222 Rosewood Drive, Danvers, MA 01923, USA. In this case permission to photocopy is not required from the publisher.

ISBN 978-1-78326-363-9 


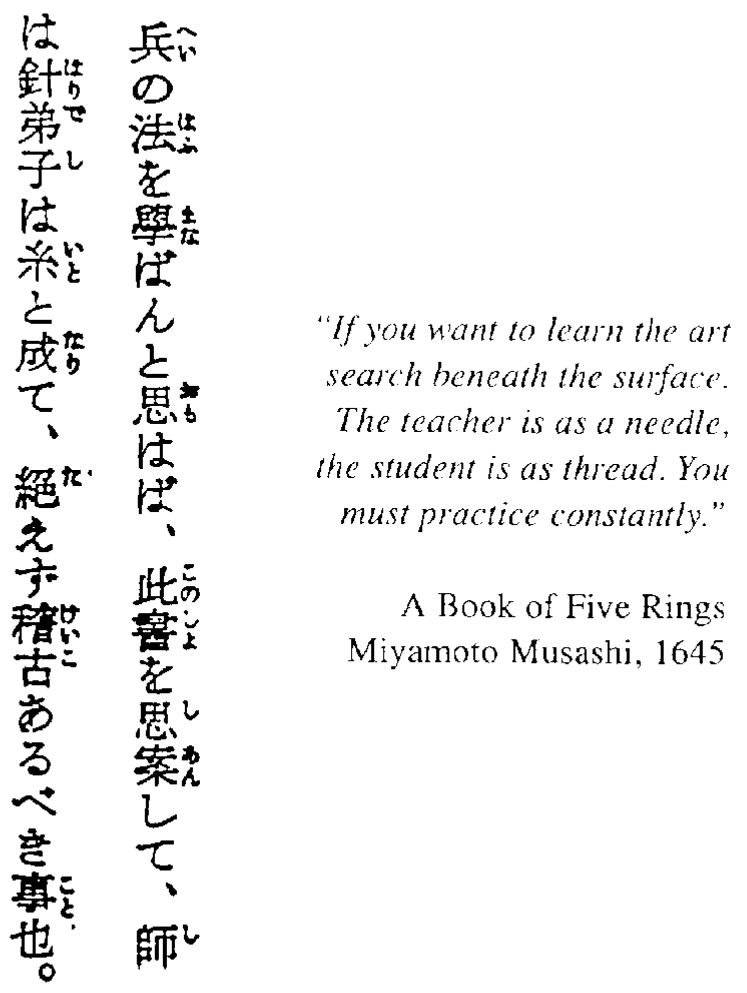


This page intentionally left blank 


\section{Preface to the Third Edition}

Since publishing the second edition of this book, I have been very much involved with examining the nature of mode particle interactions in toroidal confinement devices. This work has resulted in a large extension of the chapter on the theory of mode-particle interactions. There is also a full chapter devoted to the use of lithium as a particle sink either on the plasma wall or on the divertor. If the use of lithium turns out to be worth even a small fraction of its theoreticaly possible utility, it will greatly help the program. The development of a liquid lithium divertor is presently being carried out in China. Research on its use is also being carried out at Princeton, Frascati, and Padova.

Recent good news is the confirmation of the plans to proceed with the construction of IGNITOR, partly constructed in Italy and planned to be built at the trinity site at Troitsk, near Moscow. An important missing part of the experimental data to date is information obtained from the observation of an ignited burning plasma, or at least one in which the fusion energy is an important part of the plasma heating.

A section on nonlocal transport has been added, since it has increasingly become apparent that simple local descriptions of transport using locally derived diffusion operators are not sufficient to describe transport in toroidal devices. This is particulary apparent in the reversed field pinch at Padova, since many discharges consist of magnetic field structures that are above the stochastic threshold.

There is as well a new chapter deriving equations for full cyclotron motion in a toroidal system and the comparison of some results with guiding center simulations. In some present devices, in particular in NSTX, the 
cyclotron radius is very large, leading to some question concerning the use of guiding center equations to describe particle motion.

The presentation is necessarily not uniform; the sections treating the derivation of basic magnetohydrodynamic equilibrium and stability properties, and of particle orbits, are more mathematical, whereas other sections are more approximate and descriptive in nature. This is to provide a basic foundation and at the same time arrive at a description of current phenomena without becoming overwhelmed by minute detail.

\author{
Roscoe White \\ Princeton Plasma Physics Lab \\ Princeton University, 2013
}




\section{Preface to the Second Edition}

In the intervening years since the first edition of this book was published the field of magnetically confined fusion research has made great advances, and the goal of an inexpensive, abundant, clean, and inexhaustable energy source is tantalizingly close. The record value of fusion power produced in research reactors has increased even faster than the memory capacity of semiconductor chips, doubling approximately every year. The large tokamak at Princeton, TFTR, reached fusion power output greater than one fourth of the energy input, producing 11 Megawatts of fusion power, and the joint European tokamak in England, JET, passed the 60 percent mark, producing 16 megawatts of fusion power. Both JET and the Japanese reactor JT-60, operating without tritium, approached the equivalent break even point, i.e. in the same discharge; with tritium present, they would have produced as much power as used to heat the discharge. Research tokamaks regularly produce temperatures ten times hotter than the interior of the sun, and sustained stable operation at lower temperatures has been maintained for over an hour. In specially configured discharges in DIII-D at General Atomics the transport was reduced to the theoretical minimum neoclassical limit by using sheared rotation to stabilize the turbulence. Most of the rapid large scale instabilities impeding confinement have been understood and dominated, and research focuses on improving and extending the operating domain by the control of fine-scale instabilities.

At the same time the field experienced an often rancorous debate concerning the wisdom of constructing a large multibillion dollar demonstration reactor capable of igniting, producing net power, and testing associated engineering components. The reactor, ITER, was designed as a joint effort by 
the four partners, Europe, the Soviet Union, Japan, and the United States. The debate was complicated by the collapse of the Soviet Union and the withdrawal of the United States from all but token theoretical analysis due to congressional pressure to balance the US budget. ITER is now being built in Cadarache, France, and China, India and South Korea are also participating. In addition, large independent programs are underway in Japan, China and South Korea.

Many US physicists feel that a reactor produced following the design of ITER cannot be economically successful, and the US program, aside from an official but small support for ITER, has retreated to a search for a "smaller, cheaper, smarter" solution. One such approach was guided by the discovery of a large class of stellarators, the so called quasi-symmetric stellarators, which could offer distinct advantages over tokamaks provided they succeed in obtaining high beta and reasonable confinement. The parameter space of aspect ratio, elongation, and cross-sectional shape is being explored, as is the use of various means of profile control to produce regimes of very good confinement. The idea of covering the plasma-facing toroidal walls with flowing liquid metal, to eliminate the problems associated with wall erosion and the design of divertors. An important missing experimental step is the production of a burning, ignited plasma, and most physicists agree that such an experiment is crucial. Aside from ITER, the only design for an ignited plasma device to proceed beyond paper studies is the IGNITOR, partly constructed in Italy, but its future completion is uncertain.

In spite of the discord concerning the research strategy, the field is rather optimistic. The impressive list of successes obtained in the last decades leads many research fusion scientists to believe that a good solution is "out there" waiting to be found, and that it will be found. In the meantime, relatively cheap petroleum and natural gas, along with the development of renewable energy sources continue to suppress the urgency for arriving quickly at an economical design. The minimum commitment allowing preservation of the results established so far is to continue to train and maintain a cadre of professionals capable of carrying out the search, and of rapidly producing a good reactor when such a design is found.

The book has been extended in several ways. Many of the derivations have been improved. Wherever possible the analysis has been extended to include other toroidal devices as well as tokamaks, including the analysis of equilibrium, particle orbits and stability. The area of field stochasticity and the onset of chaos has been improved. The treatments of bootstrap current, 
stochastic ripple loss, and phase integral methods have all been rewritten and extended, and new sections added treating the classification of particle orbits in tokamaks, TAE modes and their saturation, neoclassical tearing, and general wave-particle interactions.

The second edition has bendfited from comments and corrections by Mike Beer, Alain Brizard, Yanlin Wu, Wonchull Park, Don Monticello, Allan Reiman, Wei Li Lee, Zhihong Lin, Rob Goldston, Paul Parks, Stuart Zweben, Leonid Zakharov, and many others, as well as the availability of LATEX word processing. Many of my students have contributed by posing good questions and by showing interest in the further development of some topics.

Roscoe White

Princeton Plasma Physics Lab

Princeton University, 2001 
This page intentionally left blank 


\section{Preface to the First Edition}

These notes accompany a graduate course taught at Princeton, designed to provide a basic introduction to plasma equilibrium, particle orbits, transport, and those ideal and resistive magnetohydrodynamic instabilities which dominate the behavior of a tokamak discharge, and to develop the mathematical methods necessary for their theoretical analysis. Primarily the book covers the consequences of ideal and resistive magnetohydrodynamics, these theories being responsible for most of what is well understood regarding the physics of tokamak discharges. No attempt is made to discuss the derivation of this formalism, this being a topic better left to a course devoted to kinetic theory. The focus is rather on the description of equilibria, the linear and nonlinear theory of large scale modes, and single particle guiding center motion, including simple neoclassical effects. Modern methods of general magnetic coordinates are used, and the student is introduced to the onset of chaos in Hamiltonian systems in the discussion of destruction of magnetic surfaces. The Hamiltonian formulation of guiding center motion provides another glimpse of modern methods in particle dynamics, preparing the way for the study of gyrokinetics. The interaction of a high energy particle component with a background magnetohydrodynamic plasma, of interest for the description of intensely heated and ignited plasmas, is also treated.

Much of the book is devoted to the description of the limitations placed on operating parameters given by ideal and resistive modes, and current ideas about how to extend and optimize these parameters. This permits the student to quickly arrive at the research level of a topic which is reasonably well developed and plays an important role in our current understanding of 
the dynamics of basic confinement behavior. The part of the book dealing with transport consists of an elementary introduction to the principle neoclassical mechanisms, and examples of the perturbation methods employed to deduce transport rates from the drift kinetic equation. There is a brief introduction to some of the primitive theories and phenomenological descriptions of anomalous transport. Particle loss due to symmetry breaking perturbations such as toroidal field ripple is treated. The last chapter is a treatment of the method of phase integrals, which has proved very valuable in the study of ballooning modes, parametric instabilities, and microinstabilities. In spite of its elegance and utility there is no treatment in the literature easily accessible to the student.

To a large degree the book is self contained, and most chapters depend logically on material developed previously. The exceptions are the chapter on phase integral methods, which is independent, and the chapter on transport, which requires only Chap. 1 through 3 . The course is aimed at second year graduate students, but by changing emphasis on the subjects presented, it could also be given to first year students, or even to undergraduates with some previous plasma physics training. At the same time, the material in each topic can be easily expanded to make contact with current research, and the course could be taught at a more advanced level by using the text as a base. Notation is uniform and corresponds to that most commonly used among working plasma theorists.

Roscoe B. White Princeton 1989 


\section{Acknowledgement}

The material for these lectures has come into being during interaction with many colleagues, both at Princeton and elsewhere. The subject matter necessarily reflects my interests and the interests of the people with whom I have worked. Particularly important were Paul Rutherford, who suggested the original outline of topics when I first taught the course and provided notes for some sections, Harry Mynick and Allen Boozer in the fields of guiding center motion and magnetic coordinates, Morrell Chance and Russell Kulsrud for magnetic coordinates and ideal MHD, Liu Chen, Jim Strachan, and Rob Goldston on the fishbone mode, and Marshall Rosenbluth, Don Monticello, Wonchull Park, and Kevin McGuire on resistive instabilities. I am indebted to Francis Troyon, James Van Dam, and Burton Fried for valuable suggestions regarding the text.

The second and third editions benefited from comments and corrections by Mike Beer, Alain Brizard, Yanlin Wu, Wonchull Park, Don Monticello, Allan Reiman, Wei Li Lee, Zhihong Lin, Rob Goldston, Paul Parks, Stuart Zweben, Leonid Zakharov, and many others, as well as by the availability of Latex word processing. The third edition has also benefited from my longtime interaction with Gianluca Spizzo and other physicists at the Reversed Field Pinch experiment in Padova, and close collaboration with Leonid Zakharov, who has carried out most of the theoretical analysis of the effect of lithium-coated walls and divertors, and many discussions with Stuart Hudson. Many of my students and also students of Zhihong Lin at UC Irvine have contributed by posing good questions and by showing interest in the further development of some topics. 
Thanks are due to Barbara Sarfaty and Terry Greenberg for their care and perseverence in typing the first draft of the text, and finally much gratitude to Laura and Veronica for their patience while the book was being written. 


\section{Contents}

Preface to the Third Edition vii

Preface to the Second Edition ix

Preface to the First Edition xiii

Acknowledgement $\quad$ xv

List of Figures $\quad$ xxiii

$\begin{array}{lll}\text { Chapter } 1 & \text { Toroidal Configuration } & 1\end{array}$

1.1 INTRODUCTION . . . . . . . . . . . . . . . . . . . . 1

1.2 GENERAL COORDINATES . . . . . . . . . . . . . 6

1.3 BASIS VECTORS, METRIC TENSOR . . . . . . . . 7

1.4 VECTOR OPERATORS . . . . . . . . . . . . . . . 9

1.5 MAGNETIC FIELD REPRESENTATION . . . . . . . . . . . . . . . . . . . . . . . . . 10

1.6 MAGNETIC SURFACES . . . . . . . . . . . . . . . . . . . . . 11

1.7 MAGNETIC SURFACE DESTRUCTION . . . . . . . . . . . . . . . . . . . . . . . . . . . 13

1.8 THE STANDARD MAP . . . . . . . . . . . . . . . . . . . . 22

1.9 Problems . . . . . . . . . . . . . . . . . . . . . . 29

1.10 References . . . . . . . . . . . . . . . . . . 31

$\begin{array}{lll}\text { Chapter } 2 & \text { Equilibrium } & 33\end{array}$

2.1 INTRODUCTION . . . . . . . . . . . . . . . . . . . . . 33

2.2 THE VIRIAL THEOREM . . . . . . . . . . . . . . . . . . . . . . . 34

2.3 FIELD LINE CURVATURE . . . . . . . . . . . . . . . . . . . . 35

2.4 GENERAL 3-D EQUILIBRIA . . . . . . . . . . . . 36 
2.4.1 Hamada coordinates . . . . . . . . . . . . . . . . 40

2.4.2 Boozer coordinates . . . . . . . . . . . . . . . 41

2.4.3 Cylindrical coordinates . . . . . . . . . . . . 42

2.4.4 Zakharov coordinates . . . . . . . . . . . . 42

2.5 STELLARATORS . . . . . . . . . . . . . . . . 43

2.6 AXISYMMETRIC EQUILIBRIA . . . . . . . . . . . . 46

2.7 TOKAMAK ORDERING . . . . . . . . . . . . . . 48

2.8 THE SHAFRANOV EQUILIBRIUM . . . . . . . . . . . . . . 49

2.9 CYLINDRICAL TOKAMAK EQUILIBRIA . . . . . . . . . . 55

2.10 HIGH BETA EQUILIBRIUM . . . . . . . . . . . . . 58

2.11 FLUX CONSERVING EQUILIBRIA . . . . . . . . . . . . . 60

2.12 EQUILIBRIUM SCALING . . . . . . . . . . . . . . . 61

2.13 EQUILIBRIUM TYPES . . . . . . . . . . . . . . . . 62

2.14 STEPPED PRESSSURE EQUILIBRIA . . . . . . . . . . . 63

2.15 Problems . . . . . . . . . . . . . . . . . 69

2.16 References . . . . . . . . . . . . . . 70

Chapter 3 Guiding Center Motion $\quad 73$

3.1 INTRODUCTION . . . . . . . . . . . . . . . . . . 73

3.2 LAGRANGIAN AND HAMILTONIAN FORMULATION . . 77

3.3 ORBIT TYPES IN AXISYMMETRIC EQUILIBRIA . . . . . 82

3.4 INTEGRAL INVARIANTS . . . . . . . . . . . . . . . . 93

3.5 TOROIDAL PRECESSION . . . . . . . . . . . . . . . . 96

3.6 LARGE ASPECT RATIO . . . . . . . . . . . . . . . . . . . 98

3.7 DIAMAGNETIC CURRENT . . . . . . . . . . . . . . . . 102

3.8 CONFINEMENT OF FUSION ALPHA PARTICLES . . . . . 104

3.9 MAGNETIC PERTURBATIONS . . . . . . . . . . . . . . . . 104

3.9.1 Toroidal field ripple . . . . . . . . . . . . . . . 104

3.9 .2 Flute modes . . . . . . . . . . . . . . . . 105

3.9.3 Ideal MHD modes . . . . . . . . . . . . . . . . . . . . 110

3.9.4 Kinetic Poincaré plots . . . . . . . . . . . . . . . 111

3.10 SCATTERING AND ENERGY DIFFUSION . . . . . . . . . . 115

3.11 Problems . . . . . . . . . . . . . . . . . . . . . . 118

3.12 References . . . . . . . . . . . . . . . . . . . . . . 119

$\begin{array}{lll}\text { Chapter } 4 & \text { Linear Ideal Modes } & 121\end{array}$

4.1 INTRODUCTION . . . . . . . . . . . . . . . . . . . . . . . 121

4.2 PLASMA KINETIC AND POTENTIAL ENERGY . . . . . . 126 
4.3 SELF ADJOINTNESS OF THE POTENTIAL ENERGY . . . 127

4.4 THE ENERGY PRINCIPLE . . . . . . . . . . . . . . . . . 128

4.5 CONVENIENT FORM FOR $\delta W \ldots \ldots \ldots \ldots$. . . . . . . 129

4.6 CYLINDRICAL GEOMETRY ENERGY PRINCIPLE . . . . 133

4.7 MHD INSTABILITIES IN LOW $\beta$ TOKAMAKS . . . . . . . . 137

4.8 KINK MODE . . . . . . . . . . . . . . . . . . . . 139

4.9 THE $\mathrm{M}=1$ EXTERNAL KINK . . . . . . . . . . . . . 144

4.10 THE INTERNAL KINK MODE . . . . . . . . . . . . . . . 145

4.11 BALLOONING INSTABILITIES . . . . . . . . . . . . . . 148

4.12 MAGNETIC WELL . . . . . . . . . . . . . . . . . 154

4.13 BALLOONING, SIMPLE EQUILIBRIA . . . . . . . . . . . 155

4.14 MERCIER, SUYDAM CRITERIA . . . . . . . . . . . . . . 158

4.15 BALLOONING EQUATION MODIFICATION . . . . . . . . . 162

4.16 TAE MODES . . . . . . . . . . . . . . . . . . 163

4.17 AXISYMMETRIC MODES . . . . . . . . . . . . . . . . . 164

4.18 NUMERICAL MHD SPECTRUM . . . . . . . . . . . . 167

4.19 SHAPE AND ASPECT RATIO . . . . . . . . . . . . . . 168

4.20 Problems . . . . . . . . . . . . . . . . . . . . 172

4.21 References . . . . . . . . . . . . . . . . . . . 173

$\begin{array}{lll}\text { Chapter } 5 & \text { Linear Resistive Modes } & \mathbf{1 7 7}\end{array}$

5.1 INTRODUCTION . . . . . . . . . . . . . . . . . . . 177

5.2 THE TEARING MODE . . . . . . . . . . . . . . . . 182

5.2 .1 The tearing mode $m \neq 1 \ldots \ldots \ldots 19 \ldots$

5.2 .2 The $m=1$ tearing mode . . . . . . . . . . . . . 194

5.3 THE SKIN CURRENT PROFILE . . . . . . . . . . . . . . . 201

5.4 TOROIDAL AND SHAPING EFFECTS . . . . . . . . . . 202

5.5 THE RESISTIVE SURFACE KINK MODE . . . . . . . . . . . 204

5.6 OPTIMIZED PROFILES . . . . . . . . . . . . 206

5.7 THE RIPPLING MODE . . . . . . . . . . . . . . . . . . 208

5.8 THE RESISTIVE INTERCHANGE MODE . . . . . . . . . . . 209

5.9 RESISTIVE BALLOONING . . . . . . . . . . . . . 209

5.10 DIAMAGNETIC ROTATION . . . . . . . . . . . . 211

5.11 Problems . . . . . . . . . . . . . . . . . . 215

5.12 References . . . . . . . . . . . . . . . . . 217

Chapter 6 Mode-Particle Interaction $\quad 221$

6.1 INTRODUCTION . . . . . . . . . . . . . . . . . . . . . 221 
6.2 IDEAL DISPLACEMENT $\vec{\xi}, \alpha, \delta \vec{B}$, AND POTENTIAL . . 226

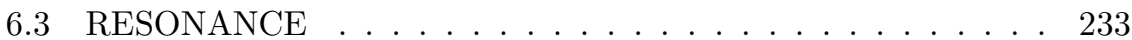

6.4 THE FIBONACCI SEQUENCE . . . . . . . . . . . . . 236

6.5 LANDAU PHASE MIXING . . . . . . . . . . . . . . . 238

6.6 PHASE VECTOR ROTATION . . . . . . . . . . . . . . 241

6.7 MODE INDUCED AVALANCHE . . . . . . . . . . . . . 246

6.8 MODE-PARTICLE ENERGY TRANSFER . . . . . . . . . . . 251

6.9 MODE EVOLUTION . . . . . . . . . . . . . . . 253

6.9.1 Frequency determined by equilibrium . . . . . . . 253

6.9.2 Frequency determined by particle distribution . . . . . . 257

6.9.3 Calculation of $\delta W_{n} \ldots \ldots \ldots \ldots$. . . . . . 276

6.9.4 Monte Carlo evaluation . . . . . . . . . . . . . 278

6.10 TAE MODE DRIVE AND SATURATION . . . . . . . . . 280

6.11 TRAPPED PARTICLE RESONANCE . . . . . . . . . . . . 284

6.12 FISHBONE INDUCED LOSS . . . . . . . . . . . . . . 287

6.13 FISHBONE DESTABILIZATION . . . . . . . . . . . . . 289

6.14 THE FISHBONE CYCLE . . . . . . . . . . . . . . . . . . 299

6.15 RESISTIVE KINETIC INTERNAL KINK . . . . . . . . . . . 302

6.16 STABILIZATION OF THE SAWTOOTH . . . . . . . . . . 303

6.17 BALLOONING DESTABILIZATION . . . . . . . . . . 308

6.18 ALPHA PARTICLE EFFECTS . . . . . . . . . . . . . . . . 309

6.19 Problems . . . . . . . . . . . . . . . . . . 311

6.20 References . . . . . . . . . . . . . . . . . . 312

$\begin{array}{lll}\text { Chapter } 7 & \text { Cyclotron Motion } & \mathbf{3 1 7}\end{array}$

7.1 INTRODUCTION . . . . . . . . . . . . . . . . . . 317

7.2 SUB CYCLOTRON HEATING . . . . . . . . . . . . . . 317

7.3 STOCHASTIC DOMAIN . . . . . . . . . . . . . . . 323

7.4 CYCLOTRON MOTION IN A TOROIDAL SYSTEM . . . . . 325

7.5 CYCLOTRON AND GUIDING CENTER ANALYSES OF RESONANCE . . . . . . . . . . . . . . . 328

7.6 PROFILE FLATTENING . . . . . . . . . . . . . . . . . 331

7.7 References . . . . . . . . . . . . . . . 333

Chapter 8 Nonlinear Behavior $\quad 335$

8.1 INTRODUCTION . . . . . . . . . . . . . . . . . . . 335

8.2 THE REDUCED EQUATIONS . . . . . . . . . . . . . . . . . 339

8.3 NONLINEAR EXTERNAL KINK . . . . . . . . . . . . . 342 
8.4 VACUUM BUBBLES . . . . . . . . . . . . . . . . . 345

8.5 NONLINEAR INTERNAL KINK . . . . . . . . . . . . . 349

8.6 COMPLETE RESISTIVE RECONNECTION . . . . . . . . . 352

8.7 NONLINEAR TEARING MODE ANALYSIS . . . . . . . . . . 352

8.8 SAWTOOTH OSCILLATIONS . . . . . . . . . . . . . 360

8.9 DISRUPTIONS . . . . . . . . . . . . . . . . . . 365

8.10 EMPIRICAL LIMITS . . . . . . . . . . . . . . . . 370

8.11 THE GREENWALD DENSITY LIMIT . . . . . . . . . . . . . 372

8.12 STABILIZATION OF TEARING MODES . . . . . . . . . 374

8.13 Problems . . . . . . . . . . . . . . . 377

8.14 References . . . . . . . . . . . . . . . . . . 378

$\begin{array}{lll}\text { Chapter } 9 & \text { Transport } & 383\end{array}$

9.1 INTRODUCTION . . . . . . . . . . . . . . . . . . . . . . . 383

9.2 THE DRIFT KINETIC EQUATION . . . . . . . . . . . . . 389

9.3 CROSS FIELD DIFFUSION . . . . . . . . . . . . . . 392

9.4 BOOTSTRAP CURRENT . . . . . . . . . . . . . . 396

9.5 NEOCLASSICAL TEARING . . . . . . . . . . . . . . . . 401

9.6 WARE PINCH . . . . . . . . . . . . . . . . . . . . . 401

9.7 MAGNETIC FIELD RIPPLE TRANSPORT . . . . . . . . . . 402

9.8 DIFFUSION IN A STOCHASTIC FIELD . . . . . . . . . . . 415

9.9 ISLAND INDUCED DIFFUSION $\ldots \ldots \ldots \ldots$

9.10 ANOMALOUS TRANSPORT . . . . . . . . . . . . . 421

9.11 CONFINEMENT SCALING . . . . . . . . . . . . 426

9.12 NONLOCAL TRANSPORT . . . . . . . . . . . . . . 428

9.12.1 Determination of the Lévy flight distribution . . . . . 432

9.12.2 Two fluid Montroll equation . . . . . . . . . . . 433

9.13 BURN CONTROL . . . . . . . . . . . . . . . . . . 435

9.14 Problems . . . . . . . . . . . . . . . . . . . 438

9.15 References ....................... . . 441

Chapter 10 The Lithium Wall Fusion Concept 447

10.1 THE IDEA OF MAGNETIC FUSION . . . . . . . . . . . . 447

10.2 CONFINEMENT REGIME CONTROLLED

BY THERMAL CONDUCTION . . . . . . . . . . . . . . 449

10.3 DIFFUSION BASED CONFINEMENT REGIME . . . . . . . 450

10.4 IMPLEMENTATION . . . . . . . . . . . . . . . . . 452

10.5 ALPHA PARTICLE CONFINEMENT . . . . . . . . . . . . 454 
10.6 DIVERTOR PLATES . . . . . . . . . . . . . . . 455

10.7 References . . . . . . . . . . . . . . . . 456

$\begin{array}{lll}\text { Chapter } 11 & \text { Phase Integral Methods } & 457\end{array}$

11.1 INTRODUCTION . . . . . . . . . . . . . . . . . . . 457

11.2 CONNECTION FORMULAE . . . . . . . . . . . . . 460

11.3 CAUSALITY . . . . . . . . . . . . . . . . . 467

11.4 BOUND STATES - INSTABILITIES . . . . . . . . . . 468

11.5 OVERDENSE BARRIER - SCATTERING . . . . . . . . . . . 472

11.6 UNDERDENSE BARRIER - SCATTERING . . . . . . . . . . . . 476

11.7 EIGENVALUE PROBLEMS . . . . . . . . . . . . . . . . . 479

11.8 Problems . . . . . . . . . . . . . . . . . . . . . . . . . 484

11.9 References . . . . . . . . . . . . . . . . . 487

$\begin{array}{ll}\text { Index } & 489\end{array}$ 


\section{List of Figures}

1.1 Fusion power output history, kilowatts. . . . . . . . . 3

1.2 General toroidal coordinates. . . . . . . . . . . . . 6

1.3 Toroidal and poloidal surfaces defining flux $\psi$ and $\psi_{p}$. . . 12

1.4 Broken flux surfaces due to a single island chain showing separatrix S, X-point and O-point. . . . . . . . . . . 14

1.5 Magnetic field structure due to perturbations. . . . . . . . . 19

1.6 Local $q_{I}$ due to magnetic islands. . . . . . . . . . . . 21

1.7 Kolmogorov entropy in stochastic bands near separatrices. . 22

1.8 Internal $q_{I}$ for the standard map vs $\epsilon$. . . . . . . . . . 24

1.9 Diffusion in the standard map. . . . . . . . . . . . . 27

2.1 A stellarator equilibrium with weak external windings. . . . 44

2.2 The W7X stellarator equilibrium. . . . . . . . . . . 45

2.3 Flux surfaces for a second order equilibrium. . . . . . . . 50

2.4 Magnetic energy and pressure in (a) low $\beta$ and (b) high $\beta$

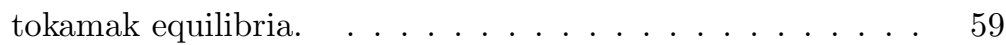

2.5 Pressure profile (smooth) from a DIIID reconstruction using STELLOPT and stepped-pressure approximation. Also shown is the $q$ profile. . . . . . . . . . . . 66

2.6 Poincaré plot of a DIIID equilibrium with perturbed boundary, calculated using SPEC, including magnetic islands and stochastic domains.

3.1 A trapped particle drift orbit. . . . . . . . . . 83

3.2 Separatrix and near separatrix orbits. . . . . . . . 86 
3.3 Orbit types in the plane of $P_{\zeta}, \mu$, for fixed high energy. . . 87

3.4 Trajectories of different orbit types, high energy. . . . . . 88

3.5 Trajectories of different orbit types, high energy. . . . . . . 89

3.6 Orbit types in the plane of $P_{\zeta}, \mu$ for fixed low energy. . . . 90

3.7 Fixed point stagnation orbits shown with dark triangles. The left branch consists of counter-passing orbits not circling the magnetic axis, situated in the inner midplane, very near the axis. The right branch consists of co-passing orbits which do not circle the axis, situated in the outer midplane. . . . .

3.8 Plane of $P_{\zeta}, E$ with $\mu B_{0}=50 \mathrm{keV}$, showing domains of confined particles. Shown are co-passing (C-P), counter-passing (CT-P), trapped (T) and trapped-loss (T-L) domains. The apexes of the parabolas are at $E=\mu B_{\min }$ (c), $E=\mu B_{0}$ (b),

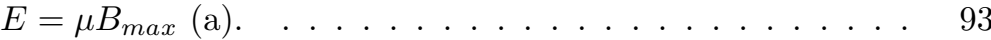

3.9 Bounce $(\kappa<1)$ and transit $(\kappa>1)$ frequency. . . . . . 100

3.10 Toroidal precession rate vs $\kappa$, with shear $s=-.2,0, .2 \ldots$. 101

3.11 Particles of gyro radius $\rho$ contributing to poloidal current. 103

3.12 Boozer (left) and equal arc (right) coordinates. . . . . . . . 108

3.13 A single harmonic $m=2, n=1$ perturbation in Boozer (left) and equal arc (right) coordinates. . . . . . . . . . . 109

3.14 Kinetic Poincaré plots for mode $m / n=9 / 3$, showing energy dependence of the $m^{\prime}=10$ resonances. . . . . . . . . . . 113

3.15 Kinetic Poincaré plots for mode $m / n=10 / 3$, showing frequency dependence of $m^{\prime}=10$ resonances. Note resonant surface unchanged from the $m=9$ mode for $58 \mathrm{kHz}$. . . . 114

3.16 Energy drag function for Monte Carlo simulations. . . . . 117

4.1 Kink mode growth rate. . . . . . . . . . . . . . . . . . 140

4.2 Current density, density, and $q$ profile for the step model. . 141

$4.3 \delta W$ for the step model. . . . . . . . . . . . . . . . . . 142

4.4 Stability diagram for kink modes. . . . . . . . . . . . . 143

4.5 Current profiles for high $m$ surface kink modes. . . . . . . . 144

4.6 A ballooning displacement. . . . . . . . . . . . . . . 149

4.7 Ballooning stability domain. . . . . . . . . . . 157

4.8 Ballooning stability including diamagnetic effects. . . . . . . 162

4.9 Alfvén waves with and without toroidal coupling. . . . . . . 164

4.10 Axisymmetric modes. . . . . . . . . . . . . . . . 166

4.11 MHD spectrum in a circular cross section cylinder. . . . . . 168 
4.12 Shaped equilibrium cross section. . . . . . . . . . . . 169

4.13 Ballooning stability domain. . . . . . . . . . . . . . 170

5.1 Geometry for resistive reconnection. . . . . . . . . . . 179

5.2 Equilibrium helical flux. . . . . . . . . . . . . . . . 183

5.3 Helical surface. . . . . . . . . . . . . . . . . . . . . . . . . 184

5.4 Magnetic field near $r=r_{s} \ldots \ldots \ldots \ldots \ldots \ldots$

5.5 Magnetic island. . . . . . . . . . . . . . . 186

5.6 Perturbations of the helical flux. . . . . . . . . . . . 187

5.7 Kruskal-Newton diagram for $m \geq 2$. . . . . . . . . . . 192

$5.8 \Delta^{\prime}$ for the peaked current profile. . . . . . . . . . . 194

$5.9 \Delta^{\prime}$ for the rounded current profile. . . . . . . . . . 195

$5.10 \Delta^{\prime}$ for the flattened current profile. . . . . . . . . . 196

5.11 Kruskal-Newton diagrams for $m=1 \ldots \ldots$. . . . . . 197

5.12 Flux surfaces and current density during $m=1$ tearing mode. . . . . . . . . . . . . . . . 198

5.13 Growth rate for the $m=1$ mode. . . . . . . . . . . 200

5.14 Unstable domains for a hollow profile. . . . . . . . . . . 202

5.15 The stabilizing effect of aspect ratio. . . . . . . . . . 203

5.16 The stabilizing effect of ellipticity. . . . . . . . . . . 204

5.17 Growth rate for the kink-tearing mode. . . . . . . . . . 207

5.18 A tearing mode stable profile. . . . . . . . . . . 208

5.19 Stability domain for the resitive ballooning mode. . . . . . 211

5.20 Growth rate of the kink and tearing mode branches. . . . . 214

6.1 Mirnov signal during fishbone mode, outline added by artist. 222

6.2 Internal kink mode stability diagram. . . . . . . . . . . 223

6.3 A Poincaré plot of field lines for an odd parity perturbation with $m / n=3 / 2$, with $\psi_{m, n}\left(r_{s}\right)=0$ at the rational surface $r_{s} / a=0.7$ where $q=3 / 2$. No island elliptic points form at the rational surface, but hyperbolic points do, and islands form on each side of the surface.

6.4 Fibonacci sequence for the standard map, showing in triangles the resonances and in large squares those resonances that destroy new KAM surfaces at each level up to $k=6$.

6.5 The standard map for $\epsilon=0.5$, showing the large $m=1$ islands and the first few Fibonacci islands for $m=2,3,4,5$. 
6.6 Circulation of particles within a resonance. At left is shown the Poincaré plot given by very many toroidal transits, and at right snapshots taken at fixed time intervals of the particles initially at $\theta=-.2$, showing the variation of the rotation rate as a function of distance from the O-point. . . . . . . . 240

6.7 Landau mixing model, showing resonance coordinates and mean particle energy vs time.

6.8 The $P_{\zeta}, \theta$ plane showing a single $m=1$ resonance island, and vectors between nearby points on good KAM surfaces and in the island. On nearby KAM surfaces the phase vector can rotate by at most $\pi$, whereas a phase vector in an island rotates through $2 \pi$ with a period given by the trapping bounce time.

6.9 Kinetic Poincaré plot for $2 / 1,3 / 2$, and $1 / 1$ zero frequency tearing modes, and amplitudes $\alpha=10^{-5} R$, and phase vector rotation indicator. Resonant surfaces from Eq. 6.34 are also shown with large triangles for surfaces $2 / 1,5 / 3,3 / 2,4 / 3$, and $1 / 1$, listed in order as they appear from the left in the plot.

6.10 Poloidal harmonics $\left(\times 10^{-6}\right)$ of an $81 \mathrm{kHz} n=3$ TAE mode with $10 \leq m \leq 23$, observed in DIII-D in shot 122117 and the plane of $P_{\zeta}, E$ with $\mu B_{0}=14 \mathrm{keV}$, with paths for kinetic Poincaré plots.

6.11 Poincaré plots associated with lines originating at 44 and 25 keV in Fig. 6.10.

6.12 Plane of $P_{\zeta}, E$ with $\mu B_{0}=5 \mathrm{keV}$, for a broad $10 \mathrm{kHz}$ mode with $m / n=6 / 5$ and amplitude $\alpha=5 \times 10^{-6} R$ and a kinetic Poincaré plot made along the line originating at the wall at $12 \mathrm{keV}$. Resonance locations from Eq. 6.34 are shown for $m^{\prime} / n=9 / 5,8 / 5,7 / 5,6 / 5$, and $5 / 5$, and also, at points where no islands are visible in the Poincaré plot, for $m^{\prime} / n=13 / 10$, and $m^{\prime} / n=11 / 10$.

6.13 Plane of $P_{\zeta}, E$ with $\mu B_{0}=5 \mathrm{keV}$, for a $10 \mathrm{kHz}$ local mode with $m / n=6 / 5$ and a kinetic Poincaré plot for amplitude $\alpha=5 \times 10^{-5} R$ made along the line originating at the wall at $12 \mathrm{keV}$. 
6.14 The kinetic Poincare plot of Fig. 6.13, shown in the $X, Z$ plane, showing the extent of the stochastic domain, with $\mu B_{0}=5 \mathrm{keV}$, for a local $10 \mathrm{kHz}$ mode with $m / n=6 / 5$, and the result of annealing. Shown is the initial distribution, obtained using the annealing process, with the points marked by triangles, and a distribution obtained with a direct guiding center simulation of three milliseconds, as a dashed line, plotted vs the square root of the volume, approximately equal to the minor radius. . . . . . . . . . .

6.15 A plot of the $P_{\zeta}, \mu$ plane, showing only $23 \mathrm{keV}$ particle orbits with 20 percent energy change due to the mode, for a $n=3$ flute mode at $102 \mathrm{kHz}$ (left) and a $n=4$ flute mode at 96 $\mathrm{kHz}$ (right).

6.16 Energy transfer for fixed $\mu$ due to a $60 \mathrm{kHz}$ mode with $m / n=$ $4 / 3$ and amplitude of $10^{-4}$. Domains of energy loss are shown in red, and energy gain in black.

6.17 Growth rate vs hot particle beta and the complex frequency plane for a trapped particle distribution with $\gamma_{I}>\omega_{* i} / 2$.

6.18 Stable domain vs $\omega_{* i}$, trapped particle distribution. . . . . . 271

6.19 Growth rate vs hot particle beta for a trapped particle distribution (left), and domains of stability (s), kink (k), and fishbone (f) versus hot particle beta and ideal growth rate (right) . . . . . . . . . . . . . . .

6.20 Growth rate vs $\beta_{h}$ and the complex frequency plane for a trapped particle distribution with $\gamma_{I}<\omega_{* i} / 2$. The kink mode branch (a) and the fishbone branch (b) are shown.

6.21 Convergence of $\delta W_{n}$ in particle number, $\beta_{h}=1 . \quad \ldots . .274$

6.22 Contributions to the dispersion relation term $\delta W_{n}$ vs $\epsilon$ (left) and $\delta W_{n}$ vs $\rho / R$, (right) for $\beta_{h}=1 \ldots \ldots \ldots . \ldots 275$

6.23 Stable domain $\delta \hat{W}$ for a uniform pitch Maxwellian distribution. The left figure shows stable (s), kink (k) and fishbone (f) domains. On the right are shown the dependence of the contributions on $\beta_{h}$.

6.24 Particle distribution modification by TAE mode.

6.25 Particle loss by TAE resonance through transition from a counter-passing orbit to a banana loss orbit.

6.26 TAE induced drift island vs minor radius, showing the resonance island as well as the outward shift of the orbits. 
6.27 Neutral beam injection geometry. . . . . . . . . . . . . 287

6.28 Injected beam precession rate vs minor radius. . . . . . . . 288

6.29 PDX beam deposition. For near perpendicular injection $\left(R_{T}=36 \mathrm{~cm}\right)$ the particles are in stagnation orbits, and only for more parallel injection are there trapped and co-passing orbits.

6.30 Trajectory of an ejected beam ion, initially a stagnation orbit, and a fishbone resonance showing the large $m=1$ island produced in the particle orbits. . . . . . . . . . . 290

6.31 Nyquist contour and $D_{k}$ along real axis. . . . . . . . . . 297

6.32 Map of Nyquist contour. . . . . . . . . . . . . . . . . . . 298

6.33 Growth for trapped particle and MHD branch. . . . . . . . 300

6.34 Fishbone cycle. . . . . . . . . . . . . . . . . . . 301

6.35 Complex frequencies and growth rate, as a function of trapped particle density, for minority heating in JET. . . . . . . . 306

6.36 Stability domain for sawtooth and fishbone modes. . . . . . 307

7.1 Poincaré section for $C_{0}=1, S_{0}=0, \omega=1 / 2 \ldots \ldots 320$

7.2 Poincaré section for $k^{2} \Phi_{0}=0.1, \omega=1 / 3 \ldots \ldots \ldots$. . . . 323

7.3 Stochastic domain for $\omega=1 / 4$, with $k^{2} \Phi_{0}=0.77 . \quad \ldots . \quad 324$

7.4 Variation of the extent of the heating domain in $k \rho$ vs wave frequency for $k^{2} \Phi_{0}=0.36(\mathrm{a}), 0.8(\mathrm{~b})$, and 2.6 (c). . . . . 325

7.5 The onset of chaos at large wave amplitude as a function of

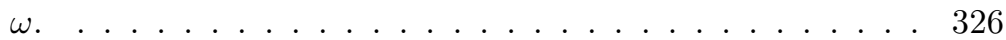

7.6 A banana trapped particle and the guiding center trajectory. 327

7.7 Resonance location for deeply trapped particles, $\mu B=0.8 E$, left, and deeply passing particles, $\mu B=0.04 E$, right. $\quad \ldots \quad 328$

7.8 Island produced in guiding center coordinates, $\rho=36 \mathrm{~cm}$ $\mu B=.04 E$.

7.9 Cyclotron orbit, $\rho=36 \mathrm{~cm}, \mu B=0.04 E . \quad \lambda=0.99$, and Poincaré plot. . . . . . . . . . . . . . 330

7.10 Cyclotron orbit, $\rho=36 \mathrm{~cm}, \mu B=0.8 E . \quad \lambda=0.6$, and Poincaré plot. . . . . . . . . . . . . . . 331

7.11 Profile using guiding center code, $\mu B=0.04 E, \mu B=0.8 E . \quad 331$

7.12 Profile using cyclotron code, $\mu B=0.04 E, \mu B=0.8 E . \quad$. . 332

8.1 Energy of the bubble state. . . . . . . . . . . . 346

8.2 Energetically favorable domain for bubble formation. . . . . 348 
8.3 Bubble evolution. . . . . . . . . . . . . . . . . . . 349

8.4 Final bubble states, low shear. . . . . . . . . . . . 350

8.5 Final bubble states, high shear. . . . . . . . . . . . . 351

8.6 Flux surface evolution during $m=1$ reconnection. . . . . . 353

8.7 Helical flux, $q$ profile and current profile before and after a Kadomtsev $m=1$ reconnection. . . . . . . . . . . . . . . 354

8.8 Numerical simulation of sawtooth crash. . . . . . . . . . . 355

8.9 Magnetic surfaces for a large $m=1$ island. . . . . . . . . 356

8.10 Island saturation width normalized to minor radius, for $m=$ 2 mode. . . . . . . . . . . . . . . 359

8.11 Sawtooth X-ray signals. . . . . . . . . . . . . . . 361

8.12 Helical flux, $q$ profile, and current profile before and after $m=1$ reconnection. . . . . . . . . . . . . . 362

8.13 Reconnection leaving $q(0)<1$. . . . . . . . . . . . 364

8.14 Soft X-ray signals during a major disruption. . . . . . . . 366

8.15 Island saturation width vs $q(0) \ldots \ldots \ldots$. . . . . . 367

8.16 Magnetic island evolution. . . . . . . . . . . . . 367

8.17 Domains of $2 / 1,3 / 2$ overlap. . . . . . . . . . . . . . 368

8.18 High beta disruption. . . . . . . . . . . . . . . . . 369

8.19 Representation of single lobe of a magnetic island schematically showing the heat flow from the auxiliary heating around the island (red arrows), the resistive heating inside the island (blue area), and the radiation losses from within the island interior (green arrow).

9.1 Neoclassical diffusion. . . . . . . . . . . . . . . . . 395

9.2 Banana orbits originating at $\theta=0$ with opposite pitches. . 396

9.3 Particle density vs pitch at the midplane. Particles with pitch $-\sqrt{r / R}<\lambda<\sqrt{r / R}$ are trapped. There is a greater density of co-moving trapped particles than counter-moving. Pitch angle scattering then produces a uniform passing distribution in $\lambda$, as shown by the dashed lines. . . . . . . . 397

9.4 Bootstrap current simulation. . . . . . . . . . . . . . 399

9.5 Magnetic field strength variation with ripple. . . . . . . . 403

9.6 A typical ripple-trapped loss orbit. . . . . . . . . . . . . . 404

9.7 Ripple well domain in Tore Supra (shaded). . . . . . . . . . 405

9.8 Modification of neoclassical diffusion due to superbanana orbits. . . . . . . . . . . . . 406 
9.9 Trapped particle trajectory showing precession resonance. The locations of the field coils are denoted by the boxes with X inside. . . . . . . . . . . . . . . . . . . 410

9.10 Poincaré plot showing ripple resonances. . . . . . . . . . . 412

9.11 Stochastic loss domain in INTOR. . . . . . . . . . . . . . 413

9.12 Fraction of alpha particles lost vs $q$ at the limiter. . . . . . 414

9.13 Neoclassical diffusion with field perturbations. . . . . . . . . 419

9.14 Diffusion vs field perturbation amplitude S. . . . . . . . . 420

9.15 Fluctuation spectrum. . . . . . . . . . . . . . . . 422

9.16 Probability matrices: left, at $\psi_{p}=0.05(r / a=0.53)$, right $\psi_{p}=0.08(r / a=0.74)$. The vertical axis is the flight distance, and the horizontal axis is the flight time.

9.17 Collisional transfer simulation: (a) an initial load of 10,000 trapped particles is evolved through the pitch angle scattering operator at $r / a \simeq 0.25$ to determine the transfer coefficients $A$ and $B$; solid lines represent the analytical fit with the solutions of Eq. 9.100; (b) the radial profile of the (average) critical pitch $\left\langle\lambda_{c}\right\rangle$, which represents the trapped particle fraction, $B /(A+B)$.

9.18 Montroll equation results. Top left $<r^{2}>$ as a function of time; top right equilibration between trapped and passing particles; botttom left particle conservation; botom right the evolution of the density profile from an initial Dirac delta, solid=passing, dashed=trapped.

10.1 Plasma column heated by energetic NBI. (a) The case of high recycling as in present fusion devices with a strong effect of the wall on the plasma. (b) The case of a pumping wall, utilizing pumping properties of liquid lithium and making the wall invisible to the plasma.

10.2 Plasma (a) temperature and (b) density profiles in the case of conventional (black) and a pumping (blue) wall.

10.3 Two key components of LiWF: (a) NBI as the heating/corefueling method, and (b) FLiLi layer on the plasma facing surface of the target plates.

11.1 Stokes diagram for $Q=\left(z-z_{l}\right)\left(z-z_{2}\right)\left(z-z_{3}\right)^{2} /(z-z 4)$ with $z_{1}=l+i, z_{2}=-1-i, z_{3}=1-i$, and $z_{4}=-1+i$. . 
11.2 Stokes diagram for a first order turning point. . . . . . . . . 462

11.3 Stokes diagram for the Bessel function. . . . . . . . . . . . . 464

11.4 Stokes plot for the bound state problem. . . . . . . . . . . 469

11.5 Stokes plot for the bound state, $a \rightarrow b \ldots \ldots \ldots$. . . . 471

$11.6 \delta$ and $\mathrm{S}$ for the bound state. . . . . . . . . . . 472

11.7 Stokes plot for the overdense barrier. . . . . . . . . . . 473

$11.8 Q=z^{2}$, Stokes plot. . . . . . . . . . . . . 474

11.9 Phase $\delta$ for overdense scattering, $Q=z^{2}-b^{2} \ldots \ldots \ldots 475$

11.10 Stokes constant for overdense scattering, $Q=z^{2}-b^{2} \ldots .476$

11.11 Stokes plot for the underdense barrier. . . . . . . . . . 477

11.12 Underdense barrier, rotated Stokes plot. . . . . . . . . . 478

$11.13 Q=z^{2}+b^{2}$, transmission and reflection vs $b \ldots \ldots \ldots . . .479$

$11.14 Q=z^{2}$, Stokes plot. . . . . . . . . . . . 480

11.15 Stokes diagram showing two turning points. . . . . . . . . 481

11.16 Stokes structure for $K<K_{c} \ldots \ldots \ldots \ldots$. . . . . . 482

11.17 Stokes structure for $K>K_{c} \ldots \ldots \ldots \ldots \ldots$. . . . 483 